\title{
Antichiral states in twisted graphene multilayers
}

\author{
M. Michael Denner $\odot,,^{1,2}$ J. L. Lado $\oplus^{1,3}$ and Oded Zilberberg $\oplus^{1}$ \\ ${ }^{1}$ Institute for Theoretical Physics, ETH Zurich, 8093 Zurich, Switzerland \\ ${ }^{2}$ Department of Physics, University of Zurich, Winterthurerstrasse 190, 8057 Zurich, Switzerland \\ ${ }^{3}$ Department of Applied Physics, Aalto University, 00076 Aalto, Espoo, Finland
}

(Received 25 June 2020; accepted 19 October 2020; published 5 November 2020)

\begin{abstract}
The advent of topological phases of matter revealed a variety of observed boundary phenomena, such as chiral and helical modes found at the edges of two-dimensional (2D) topological insulators. Antichiral states in 2D semimetals, i.e., copropagating edge modes on opposite edges compensated by a counterpropagating bulk current, are also predicted, but, to date, no realization of such states in a solid-state system has been found. Here, we put forward a procedure to realize antichiral states in twisted van der Waals multilayers, by combining the electronic Dirac-cone spectra of each layer through the combination of the orbital moiré superstructure, an in-plane magnetic field, and interlayer bias voltage. In particular, we demonstrate that a twisted van der Waals heterostructure consisting of graphene/two layers of hexagonal boron nitride $\left[(\mathrm{hBN})_{2}\right] /$ graphene will show antichiral states at in-plane magnetic fields of $8 \mathrm{~T}$, for a rotation angle of $0.2^{\circ}$ between the graphene layers. Our findings engender a controllable procedure to engineer antichiral states in solid-state systems, as well as in quantum engineered metamaterials.
\end{abstract}

DOI: 10.1103/PhysRevResearch.2.043190

\section{INTRODUCTION}

Dirac materials have sparked vast interest in recent years, as their unique electronic properties offer a controllable setting with which to realize new states of matter [1,2], as well as engineer topological phenomena $[3,4]$. A paradigmatic example of a two-dimensional (2D) Dirac material is graphene [5,6], whose spectrum exhibits Dirac-like cones at the corners (valleys) $K$ and $K^{\prime}$ of its hexagonal Brillouin zone. The two inequivalent $K$ and $K^{\prime}$ valleys have opposite chiralities with associated quantized Berry phase [2]. Correspondingly, in a finite system of zigzag termination, these valleys are connected by topological dispersionless edge states $[7,8]$. This flat edge band has been observed experimentally in a variety of systems [9-12]. Interestingly, such Dirac materials can be considered as ideal starting points for realizing other exotic surface modes [7,13-16], by introducing proper perturbations to the Dirac cones.

A paradigmatic example of the versatility of the Dirac system consists of breaking time-reversal symmetry in the honeycomb lattice and opening up a valley-dependent mass [13]. In this situation, a topologically nontrivial bulk gap opens at the Dirac points, and the above-mentioned flat edge band develops into the chiral subgap modes of a Chern insulator, where the latter are dispersive and counterpropagating on opposite edges of the 2D material $[17,18]$. Even though this state has not been observed in solid-state graphene, its

Published by the American Physical Society under the terms of the Creative Commons Attribution 4.0 International license. Further distribution of this work must maintain attribution to the author(s) and the published article's title, journal citation, and DOI. proposal led to generalizations in other materials, such as magnetically doped topological insulators $[19,20]$ and twisted bilayer graphene [21,22], as well as in engineered emulation of Chern physics in cold atoms [23,24] and photonics [25,26].

A direct generalization of such Dirac spectrum engineering includes a perturbation with a spin- and valley-dependent mass [14], which gives rise to quantum spin Hall insulators. Here, the former flat edge band states develop into helical subgap modes [27], i.e., the bulk spectrum is gapped, but at each edge a pair of counterpropagating states with opposite spins appear. In graphene, spin-orbit coupling creates a rather small topological gap, making observation of such physics challenging. Analogous physics, however, appear in more complex materials, such as monolayer tungsten ditelluride [28].

Interestingly, antichiral edge modes appear in systems where the bulk spectrum is not gapped [16]. Here, on opposite edges of the $2 \mathrm{D}$ system, the modes propagate in the same direction, compensated by oppositely dispersive semi-bulkmodes. Such antichiral states are generated by introducing a valley-dependent energy shift to the Dirac cones, which can be mathematically engineered using tailored long-range hopping amplitudes [16,29,30]. Such hopping is not present in real graphene, and even though antichiral states are technically viable [31], solid-state realizations of them have not been found so far.

In this paper, we put forward a mechanism to create antichiral states based on graphene multilayers and applied electromagnetic fields. The system's additional layer degrees of freedom bring forth antichiral states in a realistic solidstate platform through a combination of an applied in-plane magnetic field and interlayer bias voltage. Thus Dirac cones of different layers are selectively modified, so that copropagating edge modes in opposite edges manifest. We first illustrate 
this idea in aligned bilayer sheets, providing a mechanism that can be readily applied to cold atom setups [32-34] and artificial Dirac systems [35,36]. We then extend our proposal to a graphene/two layers of hexagonal boron nitride $\left[(\mathrm{hBN})_{2}\right]$ /graphene twisted multilayer, demonstrating that the increased effective lattice constant allows for the creation of antichiral states at a magnetic field of $8 \mathrm{~T}$ for a $0.2^{\circ}$ twist rotation. Our proposal puts forward a viable scheme by which to realize antichiral states in twisted van der Waals systems, opening up future experimental studies of antichiral systems.

The paper is organized as follows: In Sec. II, we start by illustrating the fundamental mechanism on $A B$-stacked bilayer graphene, exploring the impact of in-plane magnetic fields and interlayer bias voltage on both the bulk and edge modes of the system. Specifically, we show that the in-plane magnetic field shifts the Brillouin zones of the two layers relative to one another, such that Dirac cones of different layers can couple in reciprocal space. In this setting, the added interlayer bias voltage tilts the relative energies of the two cones, revealing antichiral edge states in a finite system. In Sec. III, we demonstrate that a similar phenomenology happens in a twisted bilayer system, where the emergence of the moiré length dramatically reduces the required magnetic field. Specifically, we show that a graphene $/(\mathrm{hBN})_{2} /$ graphene twisted multilayer provides a feasible van der Waals system for the creation of antichiral states. Finally, in Sec. IV, we summarize our results and provide an outlook to our findings.

\section{ANTICHIRAL STATES IN $A B$-STACKED GRAPHENE BILAYERS}

To illustrate how antichiral states can be engineered in a stacked $A B$ graphene bilayer, let us first briefly summarize the electronic properties of graphene monolayer and aligned bilayers. Single-layer graphene is a hexagonal two-dimensional material with two atoms (denoted $A$ and $B$ ) per unit cell. As the atoms are identical (carbon), its energy spectrum exhibits massless Dirac-cone band touchings at the six corners of its hexagonal Brillouin zone. The cones appear in pairs with opposite chiralities at the so-called $K$ and $K^{\prime}$ valleys [2]. At a proper termination of the graphene system, e.g., at a zigzag termination, flat edge bands spectrally connect the two valleys. Using a coupled-wire description of the 2D system, these edge bands can be understood to be made of uncoupled $0 \mathrm{D}$ bound modes of $1 \mathrm{D}$ topological insulators [8]. Including a sublattice-dependent second-neighbor hopping [16], the Dirac cones can be shifted in energy, turning the topological flat bands into antichiral states. However, real graphene monolayers do not host such long-range hopping, and the Dirac cones are located at the same energy. As a result, such a valley-dependent energy shift must be artificially engineered.

Graphene bilayers can provide a possible platform to engineer valley-dependent energy shifts. They consist of two coupled graphene monolayers that can be arranged in three main configurations: $A A, A B$, and twisted stacking. In the following, we start by considering $A B$-stacked graphene, in which an atom of the $B_{1}$ sublattice is directly situated above an atom of the $A_{2}$ sublattice, where 1 and 2 denote top and bottom layers, respectively. The remaining $A_{1}$ and $B_{2}$ lie at the center of the honeycombs formed by the complementary layer; see
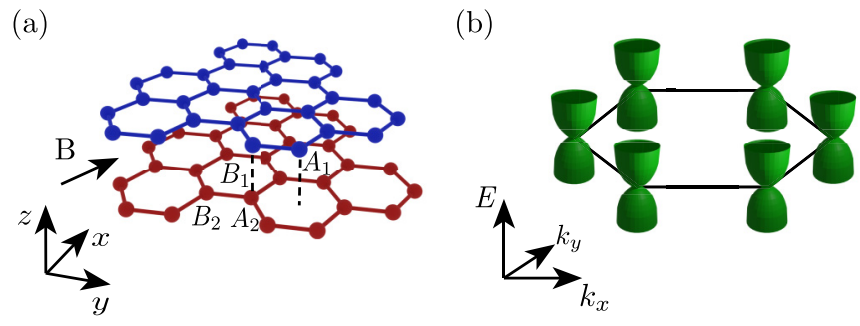

FIG. 1. $A B$-stacked bilayer graphene. (a) Real-space lattice of $A B$-stacked bilayer graphene, consisting of two graphene sheets that are shifted with respect to each other, while being aligned only at two points of the unit cell. (b) Corresponding schematic low-energy spectrum in the hexagonal Brillouin zone. The combination of the two graphene sheets results in a quadratic low-energy band structure, appearing at the six corners of the first Brillouin zone.

Fig. 1(a). The unit cell of the $A B$-stacked bilayer therefore contains four atoms. Similarly to single-layer graphene, the corresponding low-energy spectrum exhibits six band touching points divided into two inequivalent valleys. Contrary to the graphene monolayer, the spectrum in these valleys is not linear, but displays a quadratic dispersion; see Fig. 1(b) $[37,38]$. We can intuitively understand this difference: In the absence of interlayer tunnel coupling, the system has four Dirac cones (two per layer and two per valley). The interlayer coupling hybridizes the $B_{1}$ and $A_{2}$ orbitals and repels states from half filling, leading to high-energy bands. The states localized at the uncoupled $A_{1}$ and $B_{2}$ sites remain uncoupled and generate the low-energy quadratic touching points. In this bilayer case, Dirac cones between different layers can be shifted in energy simply by applying a bias between the layers, yet the already existing hybridization between cones gives rise to a gap opening [39] and the emergence of a quantum valley Hall state [40]. In the following, we will show how this gapping out of all the Dirac cones is avoided by creating momentum shifts with applied in-plane magnetic fields.

Applying an in-plane magnetic field between the two graphene layers modifies the quasimomentum of each graphene layer and is described by the minimal coupling $\mathbf{p} \rightarrow \mathbf{p}-\mathbf{A}$. The vector potential $\mathbf{A}$ incorporates the magnetic field, which can, for a general in-plane field with orientation $\phi$, be written as $\mathbf{B}=B(\sin (\phi), \cos (\phi), 0)^{\mathrm{T}}$ with $\mathbf{A}=$ $B(\cos (\phi) z,-\sin (\phi) z, 0)^{\mathrm{T}}$. Choosing the coordinate origin to coincide with a lattice site of the lower layer, we obtain that only the quasimomentum in the upper layer is affected by the in-plane magnetic field. This implies that the in-plane electron momentum in the upper layer is changed by the external field according to $\mathbf{p} \rightarrow \mathbf{p}+\Delta p$ with $\Delta p=$ $B d(-\cos (\phi), \sin (\phi), 0)^{\mathrm{T}}$, where $d$ is the distance between the two layers. Equivalently, this can be understood as a momentum kick by a Lorentz force acting on electrons tunneling between the two layers [41]. This causes the quadratic spectrum to separate back into Dirac cones of the upper and lower layer [41,42], which are now shifted in momentum with respect to each other; see Fig. 2(a). The separation of the Brillouin zones of the two layers is proportional to the strength $B$ and depends on the orientation $\phi$ of the applied magnetic field.

As the Dirac cones are shifted with respect to each other, the possibility arises to merge them once more at specific 

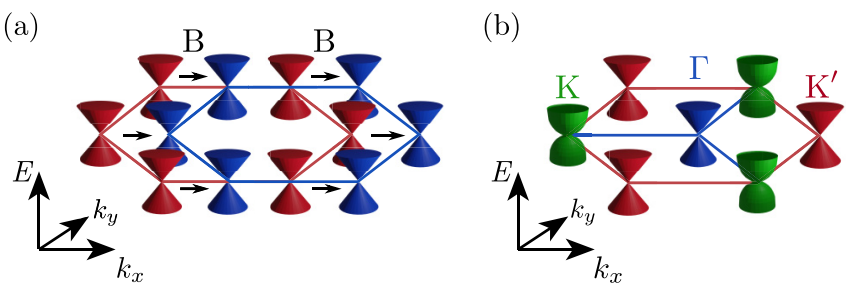

FIG. 2. $A B$-stacked graphene subject to an in-plane magnetic field. Schematic depiction of the low-energy band structure in the first Brillouin zone for (a) a small in-plane magnetic field $B$ and (b) a large $B$ that hybridizes the cones once more. The Lorentz force acting on electrons tunneling between the two graphene layers causes a momentum separation between the lower (red) and upper (blue) layer spectra [arrows in (a)], thus effectively decoupling the layer Dirac-cone spectra from one another. A strong in-plane magnetic field is able to merge Dirac cones by re-combining different valleys, thus selectively producing quadratic spectra at the merged points, while separating the band structures of the layers in other parts of the Brillouin zone.

angles and field strengths. An interesting situation occurs for a field strength of $B=\frac{1}{a d} \frac{4 \pi}{3 \sqrt{3}}$, where $a$ is the lattice constant and $d$ is the layer spacing. Here, the field strength corresponds to the distance between opposite valleys in the hexagonal Brillouin zone. Thus only one pair of valleys from the two layers is merged, whereas the other valley pair remains separate; see Fig. 2(b). Correspondingly, the merged valleys exhibit quadratic dispersion band touchings as in the $A B$-stacked case, while the valleys that are not merged exhibit linear Dirac cones as in a monolayer graphene. At the corners of the hexagonal Brillouin zone, the latter are formed by the lower layer that is unaffected by the magnetic field. At the $\Gamma$ point, the shifted upper layer's Dirac cone appears.

The effect of the magnetic field can be incorporated into a tight-binding description of the system using Peierls's substitution, i.e., by modifying the hopping amplitudes as

$$
t_{i j} \rightarrow t_{i j} e^{i \Phi_{i j}}=t_{i j} e^{i \int_{\mathbf{r}_{i}}^{\mathbf{r}_{j}} \mathbf{A} \cdot d \mathbf{r}},
$$

where $\mathbf{A}$ is the vector potential, $\mathbf{r}_{i, j}$ are the positions of the atoms in the material, and $t_{i j}$ is the bare hopping amplitude between electron valence orbits on atoms $i$ and $j$. We, thus, write down the tight-binding Hamiltonian for $A B$-stacked graphene $[37,38]$ in the presence of an in-plane magnetic field and obtain the corresponding spectrum; see Fig. 3(a). Similar to the effective low-energy description in Fig. 2, the in-plane magnetic field clearly separates the band structures of the two layers in certain areas of reciprocal space. In the nonmerged valley at the $\Gamma$ point, the spectrum originates from isolated monolayers, whereas the merged valley exhibits a quadratic dispersion caused by interlayer coupling.

By applying a bias voltage between the stacked layers, the energies of the states in each layer are shifted with respect to one another (inducing layer polarization), and a gap opens up at the quadratic touching points [43-45]. As a consequence, in standard $A B$-stacked graphene with $B=0$, the system is fully gapped, as the states forming the quadratic touching points are split off. Introducing an in-plane magnetic field $B \neq 0$, a similar behavior is obtained at the merged valley, where (a)

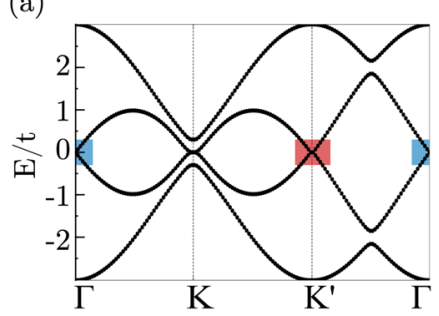

(b)

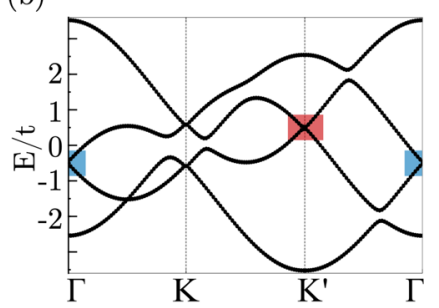

(c)

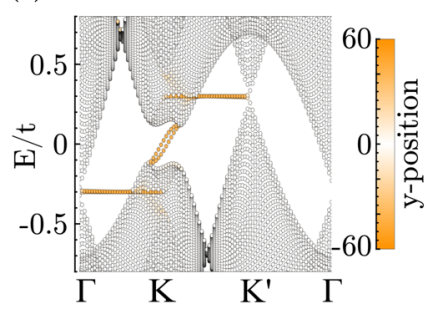

(d)

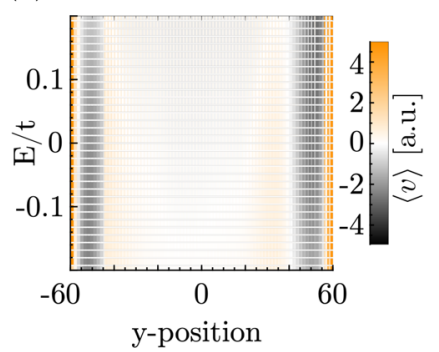

FIG. 3. Band structure of $A B$-stacked bilayer graphene. (a) The spectrum with an in-plane magnetic field that merges Dirac cones from different layers at the $K$ points (quadratic touching) and leaves linear uncoupled Dirac cones at the $\Gamma$ and $K^{\prime}$ points (marked by blue and red squares); cf. Fig. 2. (b) Since the quadratic touching points are formed by states originating from different layers, an interlayer voltage bias gaps them. Moreover, the bias voltage shifts in energy the Dirac cones of the upper and lower layers relative to one another. (c) This procedure reveals antichiral edge states when considering a finite system of 20 unit cells with zigzag termination, with the color map indicating the position of eigenstates along the finite dimension. (d) The antichiral states propagate in the same direction along the boundary (orange) compensated by a bulk current (black) of opposite direction close to the edge, as can be seen by the spatially resolved group velocity.

the quadratic dispersion is replaced by a band gap, which is tunable by the strength of the induced layer polarization; see Fig. 3(b). At the same time, since the in-plane field decoupled the spectra of the two layers at the $\Gamma$ and $K^{\prime}$ points, the full system is not gapped, but instead, the Dirac cone originating from the upper layer is shifted opposite in energy to the Dirac cone of the lower layer. In turn, this means that the system is no longer a semimetal, but it becomes a conductor, as there is a finite density of states crossing at any filling of the material.

Interestingly, at half filling, the bulk becomes conducting with a bulk current that is compensated by copropagating edge modes. The latter antichiral states are revealed in a finite system; see Fig. 3(c). A special feature of graphene is that these edge modes emerge only in certain geometries, for example, in bilayer graphene nanoribbons with zigzag edges [8,46-48]. Specifically, in our case, a finite bilayer system with zigzag termination [Fig. 3(c)] shows flat edge bands connecting the Dirac cones of each layer that are then forced to disperse due to the shifted energy between the layers. To ensure charge neutrality, this flow of charge carriers at the boundary is compensated by a bulk current flowing in the opposite direction; see Fig. 3(d). This is reflected in the bulk bands crossing half filling at the not-merged valleys at the $\Gamma$ and $K^{\prime}$ points. Note that in a perfect armchair geometry, antichiral edge states are hidden by the projection of both graphene valleys onto the same momentum. 

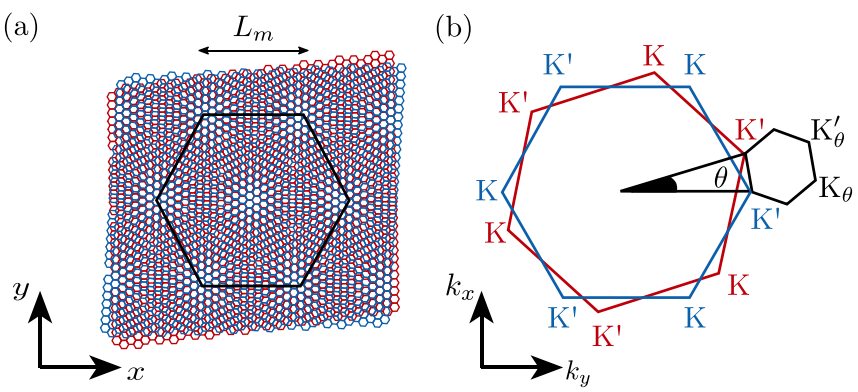

FIG. 4. Twisted bilayer graphene. (a) Real-space moiré pattern formed by the relative rotation of two graphene layers (red and blue mark atoms in the lower and upper layers, respectively). (b) Corresponding Brillouin zones of the lower (red) and upper (blue) layer rotated by angle $\theta$. The superstructure in real space of length $L_{m}$ induces a much smaller Brillouin zone in reciprocal space.

We thus obtain a scenario that generates antichiral states in a realistic system, using the tunability offered by the application of magnetic and electric fields on bilayer systems. Note that the key ingredient of shifted Dirac cones that drag in energy the topological flat edge bands manifests here in similitude to the case of the modified Haldane model [16]. Furthermore, we can reverse the propagation direction of these antichiral states either by changing the sign of the layer polarization or by using an opposite field to merge the Dirac cones at the $K^{\prime}$ valley. However, the in-plane magnetic field required to shift the stacked graphene Brillouin zones with respect to each other for a real graphene bilayer corresponds to an unrealistically large value, $B=\frac{1}{a d} \frac{4 \pi}{3 \sqrt{3}}=19.6 \mathrm{kT}$, where we converted to real units by taking $a=2.46 \AA$ [37] and $d=3.3 \AA$ [41]. Nevertheless, it is important to emphasize that such large effective fields can be achieved in quantum engineered systems, where synthetic gauge fields can be induced $[26,49]$. As in this paper we are ultimately interested in showing how to create antichiral states in solid-state materials, in the following, we show how an analogous mechanism can be realized in twisted bilayer graphene, dramatically lowering the required magnetic fields.

\section{ANTICHIRAL STATES IN TWISTED BILAYER GRAPHENE}

In the previous section, we saw that the $A B$-stacked bilayer graphene exhibits antichiral states once two coupled Dirac cones are shifted in energy relative to one another. Yet, the in-plane magnetic field required for selective coupling of these Dirac cones was too large, a feature simply related to the lattice constant and layer spacing associated with such a momentum space translation. Hence, in this section, we move to investigate a similar scenario in twisted bilayer graphene, in which the emergence of a new moiré length will dramatically lower the magnetic field required. In such a system, the two graphene layers are not perfectly aligned, but form a relative angle, creating a so-called moiré superstructure [50]. This causes an alternating pattern of $A B, A A$, and $B A$ stackings, resulting in a supercell up to 1000 times larger than in a graphene monolayer; see Fig. 4(a). Such moiré patterns are observed with scanning tunneling spectroscopy [51], and the period is described as [50]

$$
L_{M}=\frac{a}{2 \sin \frac{\theta}{2}} \approx \frac{1}{\theta},
$$

such that the size of the unit cell scales roughly as $\frac{1}{\theta}$ for small twisting angles $\theta$. The corresponding first Brillouin zone is again hexagonal but considerably smaller than that of a normal graphene layer; see Fig. 4(b) [50]. Correspondingly, this yields a reduced inter-Dirac-cone reciprocal distance

$$
\Delta K=\left|\mathbf{K}_{\theta}-\mathbf{K}_{\theta}^{\prime}\right|=\frac{4 \pi}{3 a} \sqrt{2} \sqrt{1-\cos \theta} .
$$

Hence, for small twisting angles, the twist dramatically reduces the distance between the Dirac valleys, i.e., this allows for much smaller in-plane magnetic fields necessary for the generation of selective Dirac-cone coupling and the appearance of antichiral states; cf. section above.

The tight-binding Hamiltonian describing the twisted bilayer graphene in the presence of in-plane magnetic fields can be written as [52-55]

$$
\begin{aligned}
H= & \sum_{\langle i, j\rangle} t\left(\Phi, \mathbf{r}_{i}, \mathbf{r}_{j}\right) c_{i}^{\dagger} c_{j}+\sum_{i, j} \hat{t}_{\perp}\left(\Phi, \mathbf{r}_{i}, \mathbf{r}_{j}\right) c_{i}^{\dagger} c_{j} \\
& +\Delta \sum_{i} \tau_{z}^{i i} c_{i}^{\dagger} c_{i} .
\end{aligned}
$$

The first term describes nearest-neighbor $\langle i, j\rangle$ hopping within the layers, with the hopping amplitude $t$ modified by Peierls's substitution (1). The hopping between the twisted layers $\hat{t}_{\perp}\left(\Phi, \mathbf{r}_{i}, \mathbf{r}_{j}\right)$ depends on the relative distance between the atoms on different layers and has its maximum value $t_{\perp}$ for perfect stacking. The last term describes the bias-voltageinduced layer polarization. We denote $\Phi=B a d$ to be the flux piercing the interlayer plaquette. As a reference, in graphene the parameters are [37] $t=3 \mathrm{eV}$ and $t_{\perp}=300 \mathrm{meV}$. Similar to the aligned $A B$-stacked case in the previous section, the in-plane magnetic field causes the energy spectrum to separate into two cones per valley, which are shifted by the applied field [56]. This means that their separation can be controlled by the angle $\phi$ and strength $B$ of the field. As a reference, the distance between the cones can be bridged by a field of $\Phi \approx 0.02$ in natural units, corresponding to a magnetic field of $B=162.2 \mathrm{~T}$, which is beyond experimentally feasible values.

On the bright side, by exploiting twist engineering, we have dramatically reduced the magnetic field required to merge the Dirac points in twisted graphene bilayers. Nevertheless, the field strength required is still too large for a feasible realization of antichiral states in twisted bilayer graphene at angles above $1^{\circ}$. A simple recipe to bring down the required fields is to increase the flux associated to a certain rotation angle or to decrease the interlayer hopping to conserve the Dirac cones at smaller rotation angles. Luckily, both schemes can be achieved by including a thin hBN insulating layer between the twisted graphene layers, where the effective hopping between the layers in the resulting graphene/hBN/graphene heterostructures can be tuned between 2 to $70 \mathrm{meV}$ when inserting three to one $\mathrm{hBN}$ layers [57]. In particular, taking two layers of $\mathrm{hBN}$ as spacers brings the effective interlayer hopping $t_{\perp}$ to $20 \mathrm{meV}$ [57], while preserving the original Dirac-cone spectra up to rotation angles of $0.1^{\circ}[52,54,58]$ 

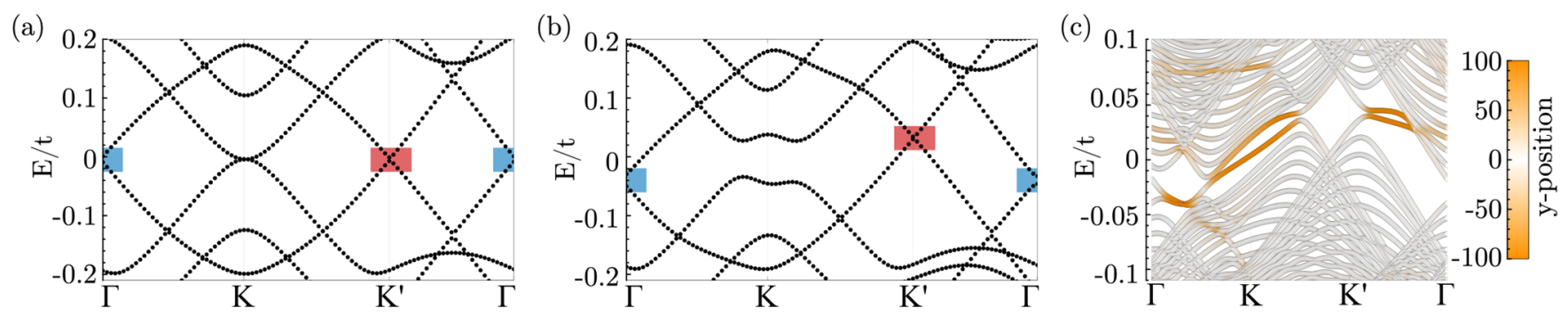

FIG. 5. Band structure of twisted bilayer graphene in the antichiral regime. (a) In the presence of an in-plane magnetic field that selectively merges Dirac cones from different layers (cf. Figs. 2 and 3), the in-plane magnetic field reduces the number of Dirac cones by creating quadratic touching points in the merged valley. (b) An applied interlayer bias voltage opens a gap at the quadratic touching points and shifts the unpaired Dirac cones from the different layers relative to one another (red and blue squares). (c) Antichiral edge states appear when considering a finite system, with the color map indicating the position of the eigenstates along the finite dimension. It is observed that the states localized on opposite edges propagate in the same direction, as expected from antichiral modes. This regime is achieved in the twisted graphene $/(\mathrm{hBN})_{2} /$ graphene heterostructure for a rotation angle of $0.2^{\circ}$ and an in-plane magnetic field of $8 \mathrm{~T}$.

(see Appendix A). Furthermore, the inclusion of two hBN layers increases the interlayer distance by approximately a factor of 3 [57]. Taking this into account, we obtain that a twisted heterostructure graphene $/(\mathrm{hBN})_{2}$ /graphene would show antichiral states for a rotation angle of $0.2^{\circ}$ and a magnetic field of $8 \mathrm{~T}$, which is an experimentally achievable regime. This demonstrates that antichiral states can be realistically engineered in twisted graphene-hBN superlattices, opening up a feasible solid-state platform for antichiral physics.

We now explicitly show that antichiral states indeed emerge in the effective model for twisted bilayer graphene. We directly focus on the regime in which the associated magnetic flux is comparable to the lattice constant, where for computational convenience we use a rescaling trick [59]. In this situation, the number of Dirac cones can be reduced by merging opposite graphene valleys by applying the required in-plane magnetic flux [Fig. 5(a)], which will be $8 \mathrm{~T}$ for the graphene $/(\mathrm{hBN})_{2} /$ graphene heterostructure at a rotation angle of $0.2^{\circ}$. In order to realize antichiral states, the two remaining Dirac cones are shifted in energy relative to one another. This is again achieved by adding an interlayer bias voltage [Fig. 5(b)], i.e., on-site energies $\Delta$ that have a different sign $\tau_{z}^{i i}= \pm 1$ depending on the corresponding layer. This causes the separated Dirac cones of the upper and lower layer to be shifted in energy, whereas the quadratic merging point is gapped out. At the gapped merging point, antichiral edge states appear. These states are expected to always appear, due to the fact that for a twisted graphene geometry it is impossible to have purely armchair edges. Since the remaining cones are shifted up and down in energy, bulk states are present at the same chemical potential that compensate the copropagating edge channels [Fig. 5(b)]. The system is no longer insulating, but metallic, with currents along the edges propagating opposite to the bulk flow [Fig. 5(c)]. To summarize, antichiral states can be obtained in a twisted bilayer graphene system using the same recipe of applying in-plane magnetic and interlayer electric fields.

We recall that in the $A B$-stacked model in Sec. II, the direction of the antichiral states could be reversed either by changing the sign of the gap-opening bias or by reversing the field direction. Interestingly, applying such a modification in the twisted case shows no change in the propagation direction. This is because of the lack of rotational symmetry of the twisted layers. Changing the signs of the on-site potentials effectively corresponds to rotating the graphene sheets around an in-plane axis, thus also changing the direction of the currents along the edges. In a normal $A A$ or $A B$ stacking, this produces the same crystal structure, with reversed layer polarization. In contrast, in twisted bilayer graphene, the angle between the two layers destroys this symmetry, such that a rotation creates a new structure, possessing identical current directions. The direction of the currents can, however, still be reversed by the time-reversal breaking direction, which corresponds to an inversion of the magnetic field. This means merging Dirac cones in a different valley. Since these valleys are related by time-reversal symmetry, the propagation direction is reversed.

\section{CONCLUSIONS}

To summarize, we put forward a procedure to create antichiral states in graphene multilayer systems, by combining magnetic fields and interlayer bias. In particular, we have demonstrated that a twisted graphene $/(\mathrm{hBN})_{2} /$ graphene heterostructure at $0.2^{\circ}$ rotation will show antichiral states for in-plane magnetic fields of $8 \mathrm{~T}$. This fundamental idea consists of engineering a system hosting two Dirac points that can be shifted in energy by means of an interlayer bias. This is achieved by shifting the Dirac cones by an in-plane magnetic field, with the regime being reached when the in-plane magnetic flux is comparable to the moire length. Besides a realistic van der Waals solid-state realization, we have also proposed a minimal system consisting of aligned graphene honeycomb lattices in which antichiral states can be created. Such a minimal scheme can be exploited in cold atom gases and engineered quantum systems. Our work therefore marks a promising step towards the realization and engineering of these special antichiral states, providing a stepping stone towards further studies in antichiral metals.

\section{ACKNOWLEDGMENTS}

We acknowledge financial support from the Swiss National Science Foundation through Grants No. PP00P2_1163818 and No. PP00P2_190078. The authors are grateful to F. Goerg, T. Wolf, and I. Petrides for helpful suggestions and fruitful discussions. J.L.L. acknowledges the computational 
resources provided by the Aalto Science-IT project and financial support from Academy of Finland Projects No. 331342 and No. 336243 .

\section{APPENDIX: HBN LAYERS AS INSULATING SPACERS AND THEIR IMPACT ON THE LOW-ENERGY BAND STRUCTURE OF THE TWISTED BILAYER GRAPHENE}

In this Appendix, we illustrate why hBN can be used for encapsulation and for studying graphene devices without strongly impacting the low-energy gapless Dirac structure [60-62]. In general, adding an hBN monolayer can have an additional impact on the electronic structure of graphene multilayers for specific angles [62]. For instance, aligning hBN with one of the twisted graphene layers [21] opens a sizable band gap in the spectrum. However, the situation is dramatically different when there is a large twist angle between the hBN and twisted graphene bilayers [62]. In such a situation, the electronic structure remains gapless, due to averaging out of the sublattice imbalance in the moire unit cell $[63,64]$. Correspondingly, a large twist angle between the hBN and the graphene multilayers allows lifting of any nontrivial impact of the $\mathrm{hBN}$ on the graphene band structure at low energies and acts as an effective spacer between the graphene layers [65]. To demonstrate this, we explicitly compute the electronic structure of a graphene/(hBN $)_{2} /$ graphene twisted heterostructure with a relative angle between the different layers of $6^{\circ}$, demonstrating that the effect of $\mathrm{hBN}$ is to effectively weaken the interlayer coupling yet without impacting the lowenergy dispersion. For this, we use an analogous real-space model as in Sec. III, but now taking layers that can have an intrinsic sublattice imbalance $m$

$$
\begin{aligned}
H= & t \sum_{\langle i, j\rangle} c_{i}^{\dagger} c_{j}+t_{\perp}^{i j} \sum_{i, j} c_{i}^{\dagger} c_{j} \\
& +m \sum_{i \in B N} \sigma_{z}^{i i} c_{i}^{\dagger} c_{i},
\end{aligned}
$$

where the first term describes nearest-neighbor $\langle i, j\rangle$ hopping within the layers, $t_{\perp}$ is the hopping between the layers, and the last term describes the intralayer imbalance between the sublattices modeled with the Pauli $\sigma_{z}$ matrix. In particular, the $\mathrm{hBN}$ layers are modeled by taking $m=1.2 t$, which gives rise to a large gap at charge neutrality. We now compute the electronic spectra of a twisted graphene bilayer that has (i) no hBN spacer between layers [Figs. 6(a) and 6(b)], (ii) two twisted hBN layers as spacers [Figs. 6(c) and 6(d)], and (iii) a vacuum spacer [Figs. 6(e) and 6(f)]. It is clearly observed that the two hBN layers as spacers yield a band structure analogous to the one with vacuum spacing, without introducing additional perturbations. This exemplifies that twisted $\mathrm{hBN}$ is capable of (a)

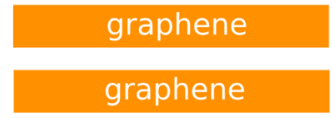

(c)

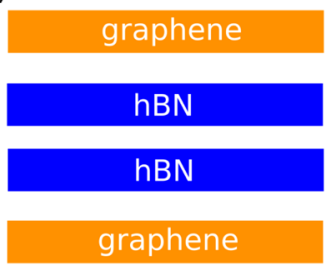

(e)

graphene

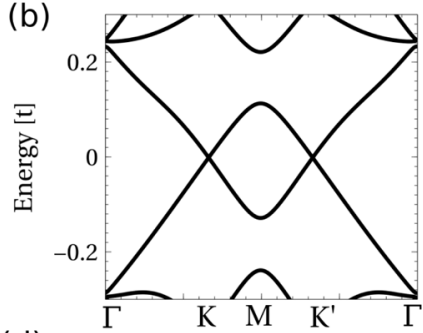

(d)

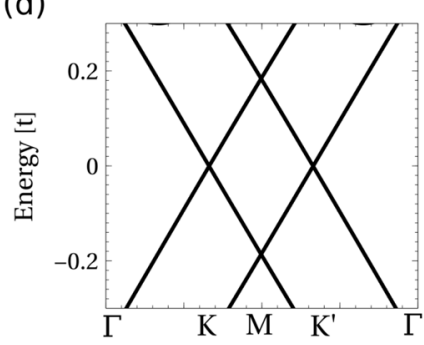

(f)

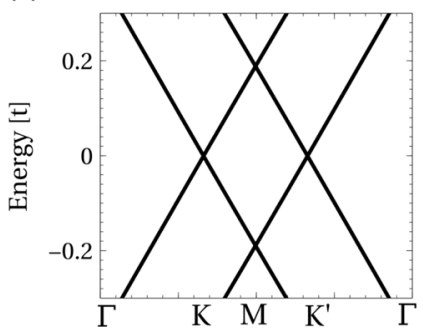

FIG. 6. Sketch (a) and band structure (b) of a twisted graphene bilayer with a conventional interlayer distance. When two twisted layers of $\mathrm{hBN}$ are put in between the graphene layers (c), the effective interlayer coupling becomes weaker (d). By comparing with a structure in which the two layers are artificially separated yielding a reduced interlayer coupling (e) it is observed that the twisted $\mathrm{hBN}$ does not create additional perturbations (f).

solely weakening the interlayer coupling in a twisted graphene bilayer, while increasing the interlayer distance. These results were performed with a full atomistic real-space tight-binding model as was Fig. 5 in Sec. III and thus incorporate all the microscopic details associated with the interatom hopping, without low-energy approximations.

Interestingly, the same effect created with an hBN spacer could be obtained by a vacuum spacing in suspended graphene devices [66-68]. These devices would also allow one to create the antichiral states proposed in our work by taking a spacing between twisted layers on the order of $1 \mathrm{~nm}$. Although suspended graphene multilayers have already been fabricated [68], suspended samples are generically more challenging to fabricate than the original graphene $/ \mathrm{hBN}_{2} /$ graphene devices we propose here.

[1] T. O. Wehling, A. M. Black-Schaffer, and A. V. Balatsky, Dirac materials, Adv. Phys. 63, 1 (2014).

[2] A. H. Castro Neto, F. Guinea, N. M. R. Peres, K. S. Novoselov, and A. K. Geim, The electronic properties of graphene, Rev. Mod. Phys. 81, 109 (2009).

[3] Y. Hatsugai, Topological aspect of graphene physics, J. Phys.: Conf. Ser. 334, 012004 (2011).
[4] M. Z. Hasan and C. L. Kane, Colloquium: Topological insulators, Rev. Mod. Phys. 82, 3045 (2010).

[5] W. Choi, I. Lahiri, R. Seelaboyina, and Y. S. Kang, Synthesis of graphene and its applications: A review, Crit. Rev. Solid State Mater. Sci. 35, 52 (2010).

[6] K. S. Novoselov, A. K. Geim, S. V. Morozov, D. Jiang, Y. Zhang, S. V. Dubonos, I. V. Grigorieva, and A. A. Firsov, 
Electric field effect in atomically thin carbon films, Science 306, 666 (2004).

[7] L. Brey and H. A. Fertig, Electronic states of graphene nanoribbons studied with the Dirac equation, Phys. Rev. B 73, 235411 (2006).

[8] P. Delplace, D. Ullmo, and G. Montambaux, Zak phase and the existence of edge states in graphene, Phys. Rev. B 84, 195452 (2011).

[9] C. Tao, L. Jiao, O. V. Yazyev, Yen-Chia Chen, J. Feng, X. Zhang, R. B. Capaz, J. M. Tour, A. Zettl, S. G. Louie, H. Dai, and M. F. Crommie, Spatially resolving edge states of chiral graphene nanoribbons, Nat. Phys. 7, 616 (2011).

[10] G. Z. Magda, X. Jin, I. Hagymási, P. Vancsó, Z. Osváth, P. Nemes-Incze, C. Hwang, L. P. Biró, and L. Tapasztó, Roomtemperature magnetic order on zigzag edges of narrow graphene nanoribbons, Nature 514, 608 (2014).

[11] S. Wang, L. Talirz, C. A. Pignedoli, X. Feng, K. Müllen, R. Fasel, and P. Ruffieux, Giant edge state splitting at atomically precise graphene zigzag edges, Nat. Commun. 7, 11507 (2016).

[12] O. Gröning, S. Wang, X. Yao, C. A. Pignedoli, G. B. Barin, C. Daniels, A. Cupo, V. Meunier, X. Feng, A. Narita, K. Müllen, P. Ruffieux, and R. Fasel, Engineering of robust topological quantum phases in graphene nanoribbons, Nature 560, 209 (2018).

[13] F. D. M. Haldane, Model for a Quantum Hall Effect Without Landau Levels: Condensed-Matter Realization of the "Parity Anomaly," Phys. Rev. Lett. 61, 2015 (1988).

[14] C. L. Kane and E. J. Mele, Quantum Spin Hall Effect in Graphene, Phys. Rev. Lett. 95, 226801 (2005).

[15] J. L. Lado, N. García-Martínez, and J. Fernández-Rossier, Edge states in graphene-like systems, Synth. Met. 210, 56 (2015).

[16] E. Colomés and M. Franz, Antichiral Edge States in a Modified Haldane Nanoribbon, Phys. Rev. Lett. 120, 086603 (2018).

[17] D. J. Thouless, M. Kohmoto, M. P. Nightingale, and M. den Nijs, Quantized Hall Conductance in a Two-Dimensional Periodic Potential, Phys. Rev. Lett. 49, 405 (1982).

[18] T. Oka and H. Aoki, Photovoltaic Hall effect in graphene, Phys. Rev. B 79, 081406(R) (2009).

[19] R. Yu, W. Zhang, H.-J. Zhang, S.-C. Zhang, X. Dai, and Z. Fang, Quantized anomalous Hall effect in magnetic topological insulators, Science 329, 61 (2010).

[20] C.-Z. Chang, W. Zhao, D. Y. Kim, H. Zhang, B. A. Assaf, D. Heiman, S.-C. Zhang, C. Liu, M. H. W. Chan, and J. S. Moodera, High-precision realization of robust quantum anomalous Hall state in a hard ferromagnetic topological insulator, Nat. Mater. 14, 473 (2015).

[21] A. L. Sharpe, E. J. Fox, A. W. Barnard, J. Finney, K. Watanabe, T. Taniguchi, M. A. Kastner, and D. GoldhaberGordon, Emergent ferromagnetism near three-quarters filling in twisted bilayer graphene, Science 365, 605 (2019).

[22] M. Serlin, C. L. Tschirhart, H. Polshyn, Y. Zhang, J. Zhu, K. Watanabe, T. Taniguchi, L. Balents, and A. F. Young, Intrinsic quantized anomalous Hall effect in a moiré heterostructure, Science 367, 900 (2019).

[23] G. Jotzu, M. Messer, R. Desbuquois, M. Lebrat, T. Uehlinger, D. Greif, and T. Esslinger, Experimental realization of the topological Haldane model with ultracold fermions, Nature 515, 237 (2014).
[24] N. Fläschner, B. S. Rem, M. Tarnowski, D. Vogel, D.-S. Lühmann, K. Sengstock, and C. Weitenberg, Experimental reconstruction of the Berry curvature in a Floquet Bloch band, Science 352, 1091 (2016).

[25] M. C. Rechtsman, J. M. Zeuner, Y. Plotnik, Y. Lumer, D. Podolsky, F. Dreisow, S. Nolte, M. Segev, and A. Szameit, Photonic Floquet topological insulators, Nature 496, 196 (2013).

[26] T. Ozawa, H. M. Price, A. Amo, N. Goldman, M. Hafezi, L. Lu, M. C. Rechtsman, D. Schuster, J. Simon, O. Zilberberg, and I. Carusotto, Topological photonics, Rev. Mod. Phys. 91, 015006 (2019).

[27] M. König, H. Buhmann, L. W. Molenkamp, T. Hughes, C.X. Liu, X.-L. Qi, and S.-C. Zhang, The quantum spin Hall effect: Theory and experiment, J. Phys. Soc. Jpn. 77, 031007 (2008).

[28] S. Wu, V. Fatemi, Q. D. Gibson, K. Watanabe, T. Taniguchi, R. J. Cava, and P. Jarillo-Herrero, Observation of the quantum spin Hall effect up to 100 kelvin in a monolayer crystal, Science 359, 76 (2018).

[29] M. Vila, N. T. Hung, S. Roche, and R. Saito, Tunable circular dichroism and valley polarization in the modified Haldane model, Phys. Rev. B 99, 161404(R) (2019).

[30] D. Bhowmick and P. Sengupta, Antichiral edge states in Heisenberg ferromagnet on a honeycomb lattice, Phys. Rev. B 101, 195133 (2020).

[31] Y. Yang, D. Zhu, Z. H. Hang, and Y. D. Chong, Observation of antichiral edge states in a circuit lattice, arXiv:2008.10161.

[32] H. M. Price, O. Zilberberg, T. Ozawa, I. Carusotto, and N. Goldman, Four-Dimensional Quantum Hall Effect with Ultracold Atoms, Phys. Rev. Lett. 115, 195303 (2015).

[33] T. Ozawa, H. M. Price, N. Goldman, O. Zilberberg, and I. Carusotto, Synthetic dimensions in integrated photonics: From optical isolation to four-dimensional quantum Hall physics, Phys. Rev. A 93, 043827 (2016).

[34] L. Tarruell, D. Greif, T. Uehlinger, G. Jotzu, and T. Esslinger, Creating, moving and merging Dirac points with a Fermi gas in a tunable honeycomb lattice, Nature 483, 302 (2012).

[35] S. Wang, D. Scarabelli, L. Du, Y. Y. Kuznetsova, L. N. Pfeiffer, K. W. West, G. C. Gardner, M. J. Manfra, V. Pellegrini, S. J. Wind, and A. Pinczuk, Observation of Dirac bands in artificial graphene in small-period nanopatterned GaAs quantum wells, Nat. Nanotechnol. 13, 29 (2017).

[36] K. K. Gomes, W. Mar, W. Ko, F. Guinea, and H. C. Manoharan, Designer Dirac fermions and topological phases in molecular graphene, Nature 483, 306 (2012).

[37] E. McCann and M. Koshino, The electronic properties of bilayer graphene, Rep. Prog. Phys. 76, 056503 (2013).

[38] A. V. Rozhkov, A. O. Sboychakov, A. L. Rakhmanov, and F. Nori, Electronic properties of graphene-based bilayer systems, Phys. Rep. 648, 1 (2016).

[39] E. V. Castro, K. S. Novoselov, S. V. Morozov, N. M. R. Peres, J. M. B. L. dos Santos, J. Nilsson, F. Guinea, A. K. Geim, and A. H. Castro Neto, Biased Bilayer Graphene: Semiconductor with a Gap Tunable by the Electric Field Effect, Phys. Rev. Lett. 99, 216802 (2007).

[40] Z. Qiao, W.-K. Tse, H. Jiang, Y. Yao, and Q. Niu, TwoDimensional Topological Insulator State and Topological Phase Transition in Bilayer Graphene, Phys. Rev. Lett. 107, 256801 (2011). 
[41] S. S. Pershoguba and V. M. Yakovenko, Energy spectrum of graphene multilayers in a parallel magnetic field, Phys. Rev. B 82, 205408 (2010).

[42] M. Van der Donck, F. M. Peeters, and B. Van Duppen, Transport properties of bilayer graphene in a strong in-plane magnetic field, Phys. Rev. B 93, 115423 (2016).

[43] E. McCann and V. I. Fal'ko, Landau-Level Degeneracy and Quantum Hall Effect in a Graphite Bilayer, Phys. Rev. Lett. 96, 086805 (2006).

[44] E. McCann, Asymmetry gap in the electronic band structure of bilayer graphene, Phys. Rev. B 74, 161403(R) (2006).

[45] H. Min, B. Sahu, S. K. Banerjee, and A. H. MacDonald, Ab initio theory of gate induced gaps in graphene bilayers, Phys. Rev. B 75, 155115 (2007).

[46] K. Nakada, M. Fujita, G. Dresselhaus, and M. S. Dresselhaus, Edge state in graphene ribbons: Nanometer size effect and edge shape dependence, Phys. Rev. B 54, 17954 (1996).

[47] M. Fujita, K. Wakabayashi, K. Nakada, and K. Kusakabe, Peculiar localized state at zigzag graphite edge, J. Phys. Soc. Jpn. 65, 1920 (1996).

[48] W. Yao, S. A. Yang, and Q. Niu, Edge States in Graphene: From Gapped Flat-Band to Gapless Chiral Modes, Phys. Rev. Lett. 102, 096801 (2009).

[49] I. Bloch, J. Dalibard, and W. Zwerger, Many-body physics with ultracold gases, Rev. Mod. Phys. 80, 885 (2008).

[50] A. V. Rozhkov, A. O. Sboychakov, A. L. Rakhmanov, and F. Nori, Single-electron gap in the spectrum of twisted bilayer graphene, Phys. Rev. B 95, 045119 (2017).

[51] R. Zhao, Y. Zhang, T. Gao, Y. Gao, N. Liu, L. Fu, and Z. Liu, Scanning tunneling microscope observations of non$\mathrm{AB}$ stacking of graphene on $\mathrm{Ni}$ films, Nano Res. 4, 712 (2011).

[52] J. M. B. Lopes dos Santos, N. M. R. Peres, and A. H. Castro Neto, Graphene Bilayer with a Twist: Electronic Structure, Phys. Rev. Lett. 99, 256802 (2007).

[53] A. O. Sboychakov, A. L. Rakhmanov, A. V. Rozhkov, and F. Nori, Electronic spectrum of twisted bilayer graphene, Phys. Rev. B 92, 075402 (2015).

[54] E. Suárez Morell, J. D. Correa, P. Vargas, M. Pacheco, and Z. Barticevic, Flat bands in slightly twisted bilayer graphene: Tight-binding calculations, Phys. Rev. B 82, 121407(R) (2010).

[55] T. M. R. Wolf, J. L. Lado, G. Blatter, and O. Zilberberg, Electrically Tunable Flat Bands and Magnetism in Twisted Bilayer Graphene, Phys. Rev. Lett. 123, 096802 (2019).
[56] Y. H. Kwan, S. A. Parameswaran, and S. L. Sondhi, Twisted bilayer graphene in a parallel magnetic field, Phys. Rev. B 101, 205116 (2020).

[57] A. Valsaraj, L. F. Register, E. Tutuc, and S. K. Banerjee, DFT simulations of inter-graphene-layer coupling with rotationally misaligned hBN tunnel barriers in graphene/hBN/graphene tunnel FETs, J. Appl. Phys. 120, 134310 (2016).

[58] R. Bistritzer and A. H. MacDonald, Moiré bands in twisted double-layer graphene, Proc. Natl. Acad. Sci. USA 108, 12233 (2011).

[59] L. A. Gonzalez-Arraga, J. L. Lado, F. Guinea, and P. SanJose, Electrically Controllable Magnetism in Twisted Bilayer Graphene, Phys. Rev. Lett. 119, 107201 (2017).

[60] C. R. Dean, A. F. Young, I. Meric, C. Lee, L. Wang, S. Sorgenfrei, K. Watanabe, T. Taniguchi, P. Kim, K. L. Shepard, and J. Hone, Boron nitride substrates for high-quality graphene electronics, Nat. Nanotechnol. 5, 722 (2010).

[61] A. F. Young, J. D. Sanchez-Yamagishi, B. Hunt, S. H. Choi, K. Watanabe, T. Taniguchi, R. C. Ashoori, and P. Jarillo-Herrero, Tunable symmetry breaking and helical edge transport in a graphene quantum spin Hall state, Nature 505, 528 (2013).

[62] J. R. Wallbank, M. Mucha-Kruczyński, X. Chen, and V. I. Fal'ko, Moiré superlattice effects in graphene/boron-nitride van der Waals heterostructures, Ann. Phys. (Berlin, Ger.) 527, 359 (2015).

[63] Y. Cao, V. Fatemi, S. Fang, K. Watanabe, T. Taniguchi, E. Kaxiras, and P. Jarillo-Herrero, Unconventional superconductivity in magic-angle graphene superlattices, Nature 556, 43 (2018).

[64] M. Koshino, N. F. Q. Yuan, T. Koretsune, M. Ochi, K. Kuroki, and L. Fu, Maximally Localized Wannier Orbitals and the Extended Hubbard Model for Twisted Bilayer Graphene, Phys. Rev. X 8, 031087 (2018).

[65] B. Amorim, J. Schiefele, F. Sols, and F. Guinea, Coulomb drag in graphene-boron nitride heterostructures: Effect of virtual phonon exchange, Phys. Rev. B 86, 125448 (2012).

[66] A. L. Grushina, D.-K. Ki, and A. F. Morpurgo, A ballistic pn junction in suspended graphene with split bottom gates, Appl. Phys. Lett. 102, 223102 (2013).

[67] Y. Nam, D.-K. Ki, D. Soler-Delgado, and A. F. Morpurgo, Electron-hole collision limited transport in charge-neutral bilayer graphene, Nat. Phys. 13, 1207 (2017).

[68] Y. Nam, D.-K. Ki, D. Soler-Delgado, and A. F. Morpurgo, A family of finite-temperature electronic phase transitions in graphene multilayers, Science 362, 324 (2018). 